In vivo-Umwandlung von Dehydroepiandrosteron-Sulfat in Östrogene

Im Verlaufe von Untersuchungen über die funktionelle Bedeutung des Dehydroepiandrosterons (DHEA) konnten wi nach intravenöser Infusion von Dehydroepiandrosteron-Phosphat (DHEA-P) eine in vivo-Umwandlung von DHEA in $\Delta^{4}$-Androsten-3,17-dion und Testosteron nachweisen ${ }^{1}$ ). Ferner wurde eine Beeinflussung des Plasmacorticosteroidspiegels unter der Einwirkung von DHEA festgestellt ${ }^{2}$ ). Eine vergleichende Untersuchung der Steroidausscheidung nach Infusion von DHEA-P, das durch körpereigene Phosphatase vollständig in freies DHEA überfühtt wird ${ }^{3}$, , und DehydroepiandrosteronSulfat (DHEA-S), ergab eine verstärkte Umwandlung von DHEA-S in Östrogene*).

Tabelle 1. Mittlere Erhöhung (d) der Ostrogene nach Infusion von Androgenen in freier Form baw. als Phosphat

\begin{tabular}{|c|c|c|c|c|}
\hline \multirow{2}{*}{ Infusion von $100 \mathrm{mg}$} & \multirow{2}{*}{$\begin{array}{l}\text { Zahl } \\
\text { der } \\
\text { Fälle }\end{array}$} & \multicolumn{3}{|c|}{ Östrogene $(\mu g / 24 \mathrm{Std}) * *)$} \\
\hline & & Ausgangslage & nach Infusion & $\Delta$ \\
\hline DHEA-P . . & 10 & 37 & 46 & 9 \\
\hline $1^{4}$-Androstendion & 8 & 27 & 42 & 15 \\
\hline Testosteron.. & 5 & 20 & 39 & 19 \\
\hline Testosteron-P. & 2 & 21 & 40 & 19 \\
\hline
\end{tabular}
Brown.

*) Summe von Östron, Östradiol und Östriol; bestimmt nach

Die mittlere Erhöhung der Östrogene nach Verabfolgung von $100 \mathrm{mg}$ DHEA ist relativ klein (Tabelle 1). Über $\Delta^{4}$-Androsten-3,17-dion und Testosteron nimmt die Umwandlungsrate $z u$. Die Zunahme der Östrogene ist bei Testosteron etwa doppelt so hoch wie bei DHEA. Diese Relationen fügen sich gut in das Biogeneseschema der Östrogenbildung ein, das von DHEA über $\Delta^{4}$-Androsten-3,17-dion und Testosteron zu Östradiol verläuft.

Tabelle 2. Erhöhung der Östrogene nach Intusion von DHEA-P und DHE $A-S$ in der angegebenen Reinentolge. Intusionsabstand: 4 Tage

\begin{tabular}{l|c|c|c|c}
\hline \multirow{2}{*}{ Fall } & Infusion von & \multicolumn{3}{|c}{ Östrogene (kg/24.Std) } \\
& 100mg DHEA & Ausgangslage & nach Infusion & $\Delta$ \\
\hline \multirow{2}{*}{1} & DHEA-P & 9 & 12 & 3 \\
\multirow{3}{*}{2} & DHEA-S & & 55 & 46 \\
& DHEA-S & \multirow{2}{*}{39} & 81 & 42 \\
& DHEA-P & & 49 & 10
\end{tabular}

Bei zwei Frauen im Alter von 23 und 30 Jahren wurde DHEA-P und DHEA-S verabfolgt (Tabelle 2). Dabei ergab sich nach DHEA-S eine 4- bis 5 fache Erhöhung der Östrogene, bezogen auf die mittlere Zunahme nach DHEA in Tabelle 1. Die Feststellung, daß DHEA-S die höchste Umwandlungsrate für Ostrogene aufweist, auch im. Vergleich zu $4^{4}$-Androsten3,17-dion und Testosteron, hebt die besondere Bedeutung des endogenen DHEA-S-Pools im Blut für die Östrogenbildung hervor.

Soeben berichten SITteri und MacDonald ${ }^{4}$ sowie BAULIEU und DRAY ${ }^{5}$ ) über eine bevorzugte Umwandlung von markiertem DHEA-S in Östrogene in der Schwangerschaft.

Deutsche Akademie der Wissenschaften zu Berlin, Institut für Mikrobiologie und experimentelle Therapie in Jena (Direktor. Prof. Dr. med. H. KNöLL), Abteilung fïr Steroidforschung

Eingegangen am 10. Februar 1964

Kurt Schubert

*) Die Steroidkonjugate wurden von Dipl.-Chem. G. Hobe und Ing.-Chem. A. KRAINHOFNER in unserer Abteilung hergestellt. Die Infusionen wurder unter der Leitung von Dr. G. BAcigalupo in der Robert-Rössle-Klinik, Berlin Buch, durchgefuhrt. Eine gemeinsame Publikation über klinische Aspekte erfolgt später.

1) Schubert, K., G. Frankenberg u. G. Bacigalupo: HoppeSeyler's Z. physiol. Chem. 336 (1964). - 2) Schubert, K., F. Rix TER u. E. HIENZSCH: Endokrinologie 45, 269 (1963). - 8) SCHUBERT, K., G. Hobe u. I. HienzSCH: Hoppe-Seyler's Z. physiol. Chem. 329, 195 (1962). - ${ }^{4}$ ) SIITERI, P.K., u. P.C. Macdonald: Steroids 2 713 (1963). - 5) Bauldev, E.-E., U. F. Dray: J. Clin. Endocrinol. and Metabolism 23, 1298 (1963).

\section{Untersuchungen zu der Steigerung der hormonabbauenden Funktion} der lädierten Leber

In der Regelung der interhormonalen Verbindung der Schilddrüse und des Eierstockes erfüllt die Leber eine wichtige Funktion. Die Hormone, die sich in diesen Drüsen bilden, beeinflussen auch die Leber. Während der Untersuchung der hepatoendokrinen Zusammenhänge beschäftigten wir uns mit der Frage: Welche Wirkung hat die Entfernung der Schilddrüse und des Fierstocks auf die östrogenabbauende Fähigkeit der Leber? Unsere Ergebnisse zeigten, daß sich diese Fähigkeit steigert, und zwar sogar in höherem MaB als bei den nur thyreoidektomierten Tieren. Bei einer anderen Untersuchung überzeugten wir uns davon, daß im hypothyreotischen $\mathrm{Zu}$ stand die kranke Leber die Östrogenbelastung sogar besser erträgt als bei gesunder Schilddrüse ${ }^{1 \mathbf{b}}$ ).

Jetzt untersuchten wir, wie die verletzte Leber die Östrogenbelastung erträgt, wenn wir die Schilddrüse und auch den Eierstock vorher entfernen. Die Ergebnisse verglichen wir mit den Östrogenbelastungswerten solcher leberlädierten Rat ten, denen wir vorher nur die Schilddrüse entfernt hatten.

Wir benutzten 98 Ratten. Bei 65 Tieren führten wir gleichzeitig Thyreoidektomie und Ovariektomie durch. 33 dienten als Kontrollen. Bei beiden Gruppen erzeugten wir während genau derselben Zeit eine Leberverletzung durch $\mathrm{CCl}_{4}$ nach KaUFMANN. Bei den zirrhotischen Ratten gaben wir zuerst 4, dann 8 Einheiten Östrogen intramuskulär.

Tabelle. Wirkung ion Östrogen auf Ratten

\begin{tabular}{c|c|r}
\hline Östrogendosis & 4 Einheiten & 8 Einheiten \\
\hline T., O. $+\mathrm{CCl}_{4}^{*}$ ). . & - & $11,60 \mathrm{Std}$ \\
n. K. $+\mathrm{CCl}_{4}$... & 63,71 & $120,15 \mathrm{Std}$ \\
T. $+\mathrm{CCl}_{4}$... . & 10,00 & $10,20 \mathrm{Std}$
\end{tabular}

*) T., O. = thyreoidektomiert und ovariektomiert. - n. K. = normale Kontrolle. $-\mathrm{T}$. = Thyreoidektomiert. $-\mathrm{CCl}_{4}=$ mit $\dot{\mathrm{CCl}}$ behandelt.

Wie die in der Tabelle zusammengefaßten Ergebnisse zeigen, sind $4 \mathrm{E}$ Östrogen auf die hypothyreotischen, leberlädierten, kastrierten Tiere unwirksam; sie lösen aber bei der nur thyreoidektomierten und leberlädierten Gruppe schon in $50 \%$ der Tiere eine Reaktion aus. Diese Reaktion beträgt aber nur etwa $1 / 6$ der Zeitdauer des Östrus bei Tieren mit gesunder Schilddrüse und Eierstock. Die doppelte Dosis löst die Reaktion schon bei den beiden operierten Gruppen aus. Ihre Zeitdauer ist aber gegenüber der Kontrollgruppe sehr kurz. Bei der hypothyreotischen, ovariektomierten Gruppe reagieren nur 55\% der Tiere, bei der nur thyreoidektomierten Gruppe dagegen $63 \%$. Diese Versuche beweisen, da 3 , wenn wir die kranke Leber von der Bindung mit Östrogenen und Thyroxin entlasten und sie auch von der Wirkung dieser Hormone befreien, die Östrogenabbindungsfähigkeit der kranken Leber wächst. Diese Angaben sind zu vollem Einklang mit den Ergebnissen der früheren Experimente. Die Hypothyreose steigert also die Östrogenabbindungsfähigkeit sogar auch dann, wenn die Leber verletzt ist1a).

Zusammenfassend können wir feststellen, daß die Ovariektomie die Leberschutzwirkung der Hypothyreose steigert. Die hypothyreotischen, kastrierten Tiere ertragen sogar nach der Leberläsion besser die Östrogenbelastung, als im Falle der intakten Hormonfunktion.

Pharmahologisches Institut (Direktor: Prof. Dr. T. VÁLYINAGY) und I. Medizinische Klinik (Divektor: Prot. Dr. B. ForNET) der Medizinischen Universität, Debrecen

Judith Kulcsár-Gergely und ANDrás Kulcsár

Eingegangen am 14. Februar 1964

1) KulCsár, A., u. J. KulCSÁR-GERGELY: Naturwissenschaften a) $49,610(1962)$; b) $50,306(1963)$.

\section{Action of an Homogenate of Rat Pituitary Gland on Mouse Regenerating Liver}

The ideas about the influence of the pituitary on liver regeneration are conflicting ${ }^{1}$ ). The present results are the first obtained from some experiments, devised bearing in mind that a probable 24-hour rhythm in hypophyseal functions $\left.{ }^{2}\right)$ could interfere with the appearance of the effects. 